\title{
Optical studies of tunneling in a magnetic field
}

\author{
J.C. Maan \\ High Field Magnet Laboratory, Research Institute for Materials, University of Nijmegen, NL-6525 ED Nijmegen, Netherlands
}

\begin{abstract}
A magnetic field creates a characteristic length for electronic motion, the magnetic length, which at high fields becomes comparable with the typical layer thicknesses of $\mathrm{MBE}$ grown heterostructures. These layer thicknesses are thin enough to allow coupling of the wavefunctions between successive layers making tunneling possible. The effect of a magnetic field parallel to the layers and perpendicular to the tunneling direction for different structures, i.e. asymmetric double quantum wells, periodic and non-periodic superlattices is reviewed.
\end{abstract}

\section{Introduction}

Tunneling is one of the most spectacular manifestations of quantum mechanics on macroscopic properties of matter. These manifestations can be studied experimentally to a high degree of detail with artificially grown semiconductor heterostructures. The strongly non-linear current versus voltage characteristics of double barrier resonant tunneling devices directly demonstrates, even at room temperature, the opening of a transmission channel for electrons when the emitter of such a device is brought into resonance with a bound state between the barriers. In this paper I want to review a few optical experiments where the effect of a magnetic field parallel to the layers of a few different types of semiconductor heterostructures is demonstrated. The devices studied are a superlattice (a periodic array of thin alternating layers of different semiconductors) a Fibonacci superlattices (a thin layered structure with thicknesses varying in a way to represent a Fibonacci sequence) and an asymmetric double quantum well (two wells of different thickness separated by a thin barrier). In the structures considered here the barrier layers are sufficiently thin to couple adjacent wells through leakage of the wave function by tunneling. The work described here has been reported elsewhere in more detail in Ref. [1] for the periodic superlattices, in Ref. [2, 3] for the Fibonacci superlattices, in Ref. [4] for the asymetric double quantum well. The basic physics for a double barrier resonant tunneling device in a magnetic field can be found in Refs. [5, 6].

\section{Energy levels in a parallel magnetic field}

The energy levels in a magnetic field for simple bands (effective mass $m^{*}$ ) in the presence of a potential variation in the $z$ direction $V(z)$ are given by the eigenvalues of the Hamiltonian

$\left[-\frac{\hbar^{2}}{2 m^{*}} \frac{\mathrm{d}^{2}}{\mathrm{~d} z^{\prime 2}}+\frac{e^{2} B^{2} z^{\prime 2}}{2 m^{*}}+V(z)\right] \phi\left(z^{\prime}\right)=E_{i}=\phi\left(z^{\prime}\right)$,

where $z^{\prime}=z+\hbar k_{y} / e B=z+z_{0}$ with $z_{0}$ the cyclotron orbit center coordinate, and $k_{y}$ the wavevector of the free particle motion in the $y$ direction. This eigenvalue equation can be solved exactly by numerical methods and in Fig. 1, the results for a system with narrow quantum wells separated by barriers of variable thickness is shown. 

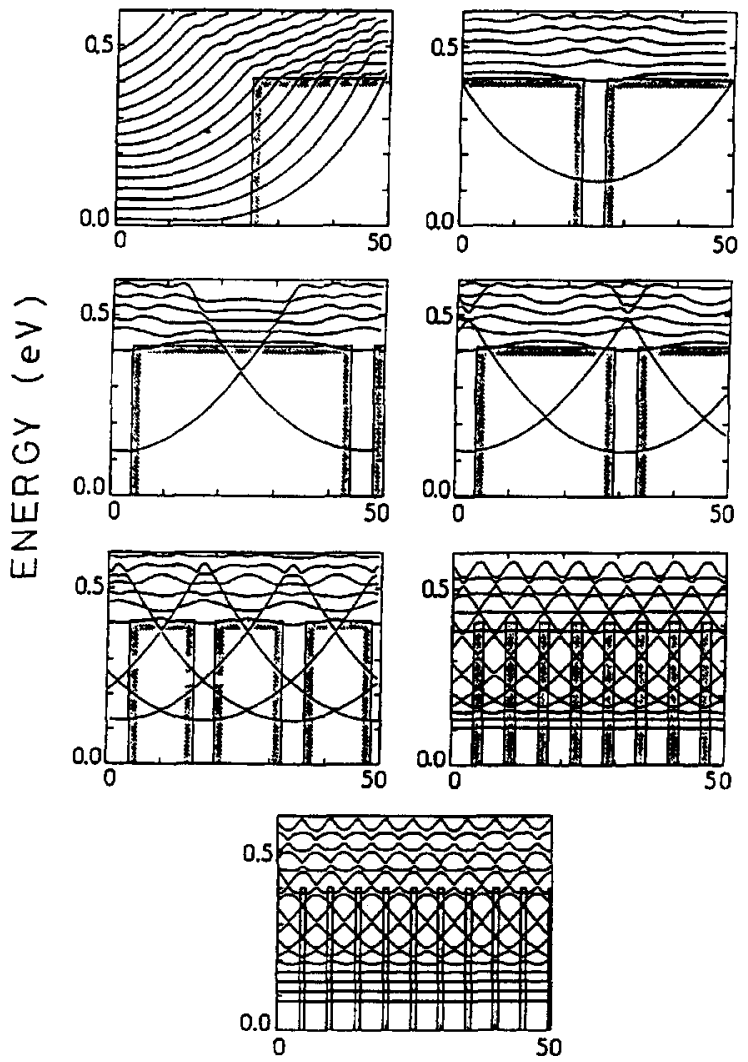

\section{CYCLOTRON ORBIT CENTER $(\mathrm{nm})$}

Fig. 1. Magnetic energy levels of a GaAs well ( $3.9 \mathrm{~nm}$ wide) in a field of $20 \mathrm{~T}$ (magnetic length $\simeq 6 \mathrm{~mm}$ ) for different barrier thicknesses as a function of the cyclotron orbit center coordinate. The flat levels develop at low energies in the superlattice miniband.

The presence of the potential $V(z)$ in Eq. (1) breaks the translation symmetry of the center coordinate $z_{0}$, i.e. it depends on the position of $z_{0}$ with respect to $V(z)$. In the absence of $V(z)$, the usual Landau levels are formed which are highly degenerate, since they have the same energy for all center orbit coordinates. This can be seen in the upper left figure where the levels at a single interface are shown and one can see that far from the step "flat" Landau levels appear. The relevant distance in this respect is the cyclotron orbit radius which for the lowest Landau level equals the magnetic length $\ell=(\hbar / e B)^{1 / 2}$.

The energy levels of a single well with width $d<\ell$ and potential $V_{0} \gg h \omega_{c}$, can be obtained by treating the magnetic field as a perturbation on the eigenvalues of the particle in a well. The perturbation expression is given by

$E=E_{n}+\frac{e^{2} B^{2}}{2 m^{*}}\left\langle z^{2}\right\rangle_{n}+\frac{1}{2 m^{*}}\left(\hbar k_{y}+e B\langle z\rangle_{n}\right)^{2}$.

Here $E_{n}$ is the subband energy of the unperturbed problem. $\left(e^{2} B^{2} / 2 m^{*}\right)\langle z\rangle_{n}$ with $\langle z\rangle_{n}$ the expectation value of $z^{2}$, is the diamagnetic shift which is small if $d_{\mathrm{w}} \ll \ell$. The last term represents the original parabolic $k_{y}$ dispersion shifted by an amount $e B\langle z\rangle_{n}=-\hbar k_{y 0}$ which depends on the position of the well in the chosen origin of the coordinate system. $\langle z\rangle_{n}$ is the expectation value of $\langle z\rangle$ and is zero for a well centered around $z=0$, but equal to $\hbar k_{y 0} / e B$ for wells centered around different values of $z$. The shift corresponds just to a shift of the cyclotron orbit center $z_{0}$, so that the lowest energy occurs when $z_{0}$ is the center of each well.

\section{Superlattices}

In Fig. 1 it can be seen that each well gives rise to a parabolic dispersion in the cyclotron orbit center coordinate as long as the wells are sufficiently far apart and tunneling between them can be neglected. For narrow barriers this is not true anymore which shows in Fig. 1 as the opening of an anticrossing gap at positions where parabola's cross each other. For very narrow barriers, this anticrossing gaps start to be so important that at low energies flat Landau levels appear again. Physically this means that tunneling has become so easy that the energy does not depend on the position anymore. Note that in Fig. 1 the scales are such that for the thinnest barriers the magnetic length extends over several periods of the superlattice which implies that completion of the cyclotron orbit involves tunneling through several barriers. It can be shown that the energy region where these flat bands occur corresponds to the miniband width of the superlattice such as can be calculated with the Kronig-Penney model [1].

This behaviour can be observed experimentally in an interband optical experiment. The flat levels give rise to a strong peak in the density of states, while the dispersive levels above the miniband edge will not correspond to a singularity in the DOS. Experimentally sharp absorption peaks are expected in the region of the flat bands which disappear at energies above the miniband width. Since the spacing between the flat levels increases with increasing field the top ones will be pushed out of the miniband width and become dispersive as the field is increased.

In Fig. 2 we show data of photoluminiscence excitation spectra on a superlattice in a parallel field. It can clearly be seen that below a certain energy sharp peaks are 


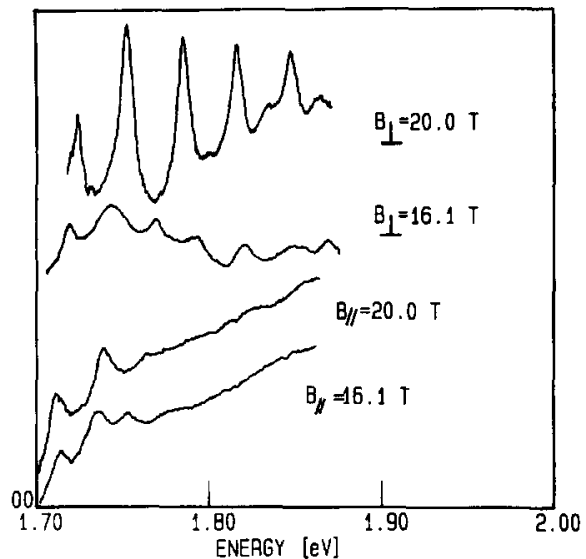

Fig. 2. Photoluminescence excitation spectra at different fields perpendicular (top) and parallel (bottom) to the layers. The lower traces show how sharp peaks disappear at the miniband edge. Sample parameters are $0.41 \mathrm{eV}$ barrier height, well and barrier thicknesses $3.9 \mathrm{~nm}$ and $1.13 \mathrm{~nm}$.

observed which disappear as soon as they are pushed out of the miniband as expected.

Similar results have also been obtained with intraband cyclotron resonance experiments [7]. The interest of these results lies in the fact that they prove that a superlattice really behaves as a coherent structure in the sense that it is not so much the fact that all individual barriers are sufficiently "transparent" to allow completion of the cyclotron orbit. In fact it is the long range periodicity which leads to the flat levels and to their disappearance at the minibands edge. This fact is completely analogous to the behaviour of any solid, where also the long range periodicity is responsible for mean free paths orders of magnitude larger than the atomic spacing.

\section{Fibonacci superlattices}

The statement of the last paragraph of Section 3 can be nicely illustrated by studying the behaviour of non-periodic superlattices. In these structures the barrier thickness $d_{\mathrm{b}}$ is equally thin but the wells have thicknesses $d_{\mathrm{w}}$ and $2 d_{\mathrm{w}}$, making the structure non-periodic. The actual sample considered was made according to a non-periodic sequence of barriers and wells

$\ldots d_{\mathrm{b}} d_{\mathrm{w}} d_{\mathrm{b}} d_{\mathrm{w}} d_{\mathrm{w}} d_{\mathrm{b}} d_{\mathrm{w}} d_{\mathrm{w}} \ldots$

constructed in such a way as to represent the 13th generation of a Fibonacci sequence [3]. The barriers are sufficiently thin so that there is a strong leakage of the wavefunctions to the adjacent wells. The energy levels in a magnetic field are shown is Fig. 3. In this case no flat

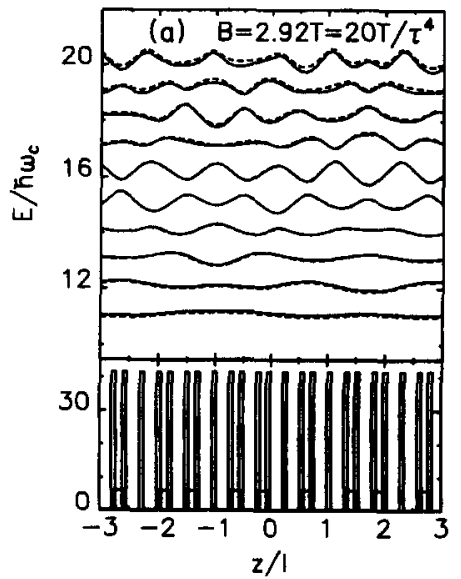

Fig. 3. Magnetic energy-level structure of a quasiperiodic superlattice at $B=2.92 \mathrm{~T}=(20 \mathrm{~T}) / \tau^{4}$ and $20 \mathrm{~T}$ as function of the cyclotron-orbit-center coordinate (top). In the lower part, a section of the Fibonacci potential at the same fields. Energies are all in units of the cyclotron energy and lengths in units of the magnetic length and the selfsimilar density of states can be observed.

Landau levels like in the periodic superlattice are observed, instead strongly dispersive, cyclotron orbit position dependent energy levels are obtained. To understand this energy level structure it is convenient to think of the potential $V(z)=0$ as a perturbation on the unperturbed free particle Landau levels. In this case $E_{N}$ is given by

$E_{N}\left(z_{0}\right)=\left\langle\phi_{N}|V(z)| \phi_{N}\right\rangle$,

with $\left\langle\phi_{N}\right|$ the harmonic oscillator wavefunction of Landau level $N$. The cyclotron orbit center dispersion, $E_{N}\left(z_{0}\right)$, reflects then the average value of the potential over the cyclotron radius as a function of the position.

In Fig. 3, the length is scaled to the magnetic length and the energy to the cyclotron energy. In these units it can be shown that [2] the center orbit dispersion at different magnetic field values which are multiples of $\tau^{2}$, with $\tau$ the golden mean $(\tau=(1+\sqrt{5}) / 2)$ have the same shape. This self similarity is a direct consequence of the self similar nature of the chosen Fibonacci potential.

Experimentally this self-similarity has indeed been observed [3] by comparing the shape of spectra at different magnetic fields. In Fig. 4 a series of spectra of Fibonacci superlattice in a parallel field is shown. Like in the periodic superlattice a Landau like levels structure is observed, as would also be expected from the calculations in Fig. 3. However, contrary to the periodic superlattice these Landau levels evolve very irregular with field, meaning that some levels are very pronounced at some field value, whereas they are almost broadened beyond recognition at other field values. Such a behaviour 
reflects the strong field dependence of the energy levels in the Fibonacci SL.

In Fig. 5, the shape of the spectra at different fields are compared to each other both for Fibonacci and for periodic superlattices. For this purpose we consider a full spectrum at a certain field $B_{0}$ and calculate the differences with a spectrum at another field $B$ with the energies all scaled to the cyclotron energy. This procedure is repeated for all other fields $B$ and the field dependence of the differences is shown in the figure. It can be seen that

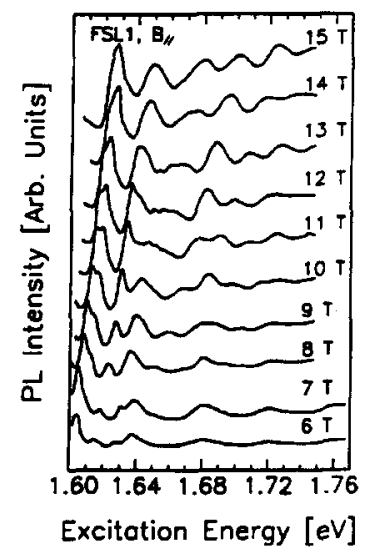

Fig. 4. PLE spectra of a Fibonacci superlattice with $d_{\mathrm{b}} \approx 1.13 \AA, d_{\mathrm{w}} \approx 1.69 \AA$ and $0.25 \mathrm{eV}$ barrier height as a function of the parallel field. Note the irregular evolution of the shape of the Landau levels with increasing field.

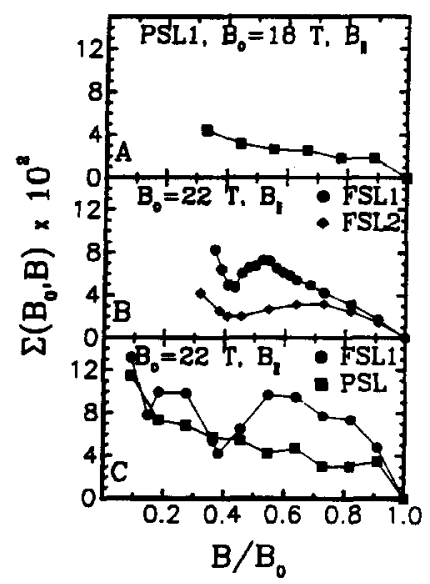

Fig. 5. Differences in shape of a spectrum at $B_{0}$ with other spectra of the same sample at a different field by scaling the energy in units of the cyclotron energy. (A) Periodic SL; (B) two different Fibonacci SL showing a minimum at $B / B_{0} \simeq 1 / \tau^{2}$; (C) theoretically calculated from the density of states. for the FSL a spectrum at given field $B_{0}$ is most similar to that at another field $B$ when $B / B_{0} \sim 0.4$. This value is very close to the expected value $1 / \tau^{2} \approx 0.38$ which directly demonstrates the property of selfsimilarity. As expected such a selfsimilarity is absent for periodic superlattices.

\section{Tunneling in an asymmetric double quantum well}

In the preceding sections we have discussed the energy level structure resulting from the coupling of adjacent wells by tunneling through a thin barrier. Here we will briefly discuss an experiment addressing more directly the tunneling process itself. The device studied is schematically shown in Fig. 6. It consists of two wells of different thicknesses (and thus different confinement energies) separated by a thin barrier. Furthermore an electric field can be applied over the whole structure so that the energy levels in the wide and the narrow well can be moved with respect to each other by the external electric field.

The experiment consists of measuring the decay time of the narrow well luminescence as a function of the applied electric field perpendicular and magnetic parallel to the layers.

If there is no other, more rapid, recombination channel, particles excited in the narrow well recombine by photon emission and the decay time is determined by the radiative lifetime. However, under flat band conditions and at zero magnetic field, electrons can also leak from the narrow well band edge to that of the wide well which is at lower energy.

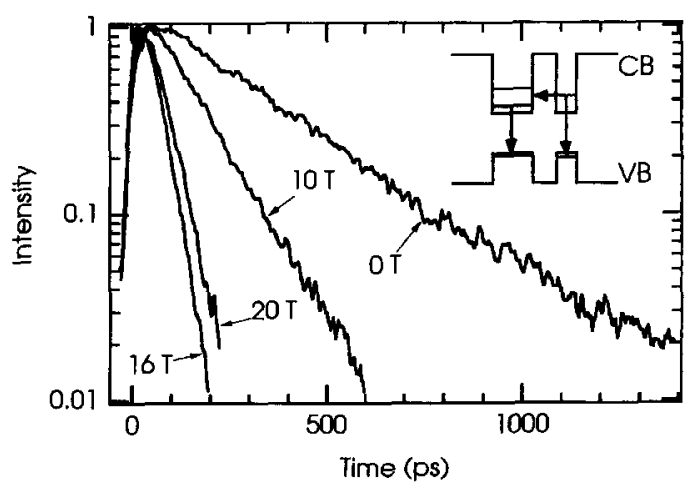

Fig. 6. Decay time of the narrow well luminescence at different parallel magnetic fields of an asymmetric double quantum well structure shown in the inset. $d_{\text {wide }}=10 \mathrm{~nm} ; d_{\text {barrier }}=6 \mathrm{~nm}$, of $\mathrm{Al}_{0.35} \mathrm{Ga}_{0.65} \mathrm{As}$ and $d_{\text {narrow }}=5 \mathrm{~nm}$ between $\mathrm{Al}_{0.35} \mathrm{Ga}_{0.65} \mathrm{As}$ cladding layers. 
This leakage cannot be a direct tunneling process since such a process involves the conservation of parallel momentum and in general there are no states with the same parallel momentum at the same energy. Resonant conditions can be created by applying a perpendicular electric field and align this way the narrow well subband with that of the wide well. In this case carriers can tunnel very rapidly to wide well, relax to the wide well band-edge and recombine by emission of luminescence at the wide-well bandgap, which is at lower energy than that from the narrow well. This process becomes visible as a drastic decrease of the narrow well luminescence decay time since at resonant conditions a new, faster, recombination channel is opened [8]. The particularity of a magnetic field parallel to the layers (and perpendicular to the tunnel current) is that in changes drastically the rules for parallel momentum conservation.

Classically this can be understood by realizing that electrons describe circular trajectories in a magnetic field so that electron motion in the $z$-direction implies a change in momentum in the $y$-direction. Therefore conservation of parallel momentum during tunneling means that states with different $k_{y}$ values in the narrow and in the wide well are connected.

Returning to our original description of the effect of a parallel field (Eq. (2)), this fact implies quantum-mechanically that the $k_{y}$ dispersion of the narrow well is shifted with respect to that of the wide well by an amount $\Delta k_{y}=(e B / \hbar) \Delta z$ where $\Delta z$ is the distance between the two centers of the wells. Therefore the two parabolas always intersect each other at a particular $k_{\text {value }}$ and for that $k$-value resonant tunneling is possible since both energy and parallel momentum conservation can be conserved.

A particular case occurs when the bottom of one parabola intersects the other at some higher energy. The condition for this is

$\Delta E=E_{\text {narrow }}-E_{\text {wide }}=\frac{\left(\hbar k_{y}\right)^{2}}{2 m^{*}}=\frac{(e \Delta z B)^{2}}{2 m^{*}}$.

By varying $\Delta E$ and $B$ and assessing where this resonance occurs, it has been shown with transport experiments that it is possible to "measure" the $E(k)$ relation. [6]

In Fig. 6, the narrow well luminescence decay time is shown as function of magnetic field. It can be seen that this time decreases drastically with increasing field and exhibits a minimum at around $16 \mathrm{~T}$. The magnetic field value of this minimum varies with the distance between $E_{\text {narrow }}$ and $E_{\text {wide }}$ which can be chosen with the external electric field. By comparison with Eq. (4), it can be shown that this minimum corresponds to the alignment of the minimum of the narrow well dispersion with the wide well parabola. From this observation it can be concluded that carriers always relax first inside the narrow well to

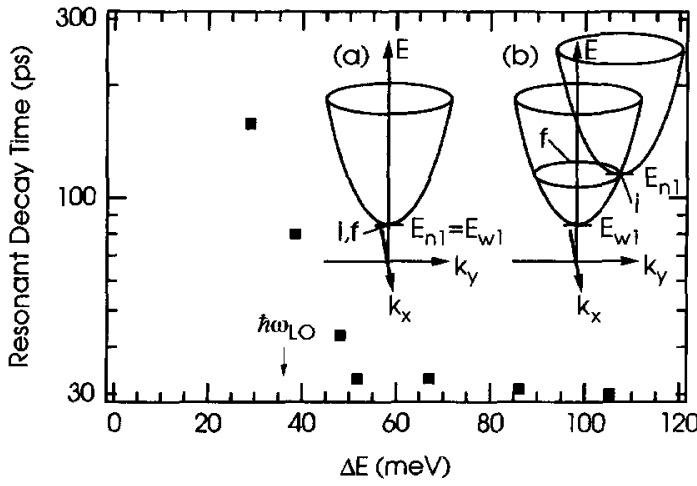

Fig. 7. Minimum luminescence decay time at the magnetically turned resonance as a function of the subband edge separation $\Delta E$. If $\Delta E>40 \mathrm{meV}$ the fast relaxation in the wide well through optical phonon emission, leads to a rapid decrease of the narrow well luminescence decay.

the band-edge and then tunnel to the wide well if this minimum is under resonant conditions.

In Fig. 7, we plot the luminescence decay time under (magnetically tuned) resonance conditions as a function of the (electrically tuned) subband separation $\Delta E$. It can be seen that the dramatic decrease of the luminescence decay time (and thus the fast tunneling from narrow to wide) is only observed as this subband separation is larger than the LO-phonon energy. A plausible explanation of this threshold energy is that through tunneling, carriers are excited at some higher energy in the wide well dispersion and that only if this energy is higher than the LO-phonon energy the narrow well "empties" rapidly through this channel. This fact can be understood by realising that if tunneling is very rapid the carriers injected in the wide well may tunnel back to the narrow well before they relax from the finite $k$ of the wide well to the bottom of the dispersion. This relaxation becomes rapid if optical phonon emission is possible $\left(\Delta E>\hbar \omega_{\mathrm{LO}}\right)$ but is too slow to prevent the back-tunneling if acoustic phonons are needed $\left(\Delta E<\hbar \omega_{\mathrm{LO}}\right)$. This observation is quite interesting since it puts clear boundaries on possible values of the actual tunneling time, i.e. faster than acoustic phonon emission but slower than optical phonon emission. Considering the controversy about tunneling times this observation could be quite significant.

\section{Acknowledgement}

The work that I have briefly reviewed in this paper is the result of a collaborative effort of many people, mentioned in the references, and whose collaboration I gratefully acknowledge. 


\section{References}

[1] J.C. Maan, Festkörperprobleme, in: Advances in Solid State Physics, Vol. 27, ed. D. Grosse (1987) 137.

[2] Y.Y. Wang and J.C. Maan, Phys. Rev. B 40 (1989) 1995.

[3] D. Toet, M. Potemski, Y.Y. Wang, J.C. Maan, L. Tapfer and K. Ploog, Phys. Rev. Lett. 66 (1991) 2128.

[4] A.P. Heberle, M. Oestreich, S. Haacke, W.W. Rühle, J.C. Maan and K. Köhler, Phys. Rev. Lett. 72 (1994) 1522.
[5] M.L. Leadbeater, L. Eaves, P.E. Simmonds, G.A. Toombs, F.W. Sheard, P.A. Claxton, G. Hill and M.A. Pate, Solid State Electron. 31 (1988) 707.

[6] R.K. Hauden, D.K. Maude, L. Eaves, E.C. Valadares, M Henini, F.W. Sheard, O.H. Hughes, J.C. Portal and L. Cury, Phys. Rev. Lett. 66 (1991) 1749.

[7] T. Duffield, R. Bhat, M. Koza, D.M. Huang, D. Grabbe and S.J. Allen Jr, Phys. Rev. Lett. 56 (1986) 2724.

[8] M.G. Alexander, M. Nido, W.W. Rühle and K. Köhler, Phys. Rev. B 41 (1990) 12295. 